\title{
LEITE HUMANO EM DIFERENTES ESTÁGIOS DE LACTAÇÃO: COMPOSIÇÃO NUTRICIONAL NO MUNICÍPIO DE CUITÉ
}

\author{
HUMAN MILK IN DIFFERENT STAGES OF LACTATION: \\ NUTRITIONAL COMPOSITION IN THE MUNICIPALITY OF CUITÉ
}

Daniel Pinheiro Fernandes ${ }^{1}$

Carolina Moreira de Santana ${ }^{2}$

\begin{abstract}
RESUMO: OBJETIVO: O presente estudo teve por objetivo analisar a composição de macronutrientes, cinza, umidade, acidez e densidade energética do leite humano de mães residentes no município de Cuité-PB durante o período de 2012-2013. MÉTODO: Trata-se de um estudo de segmento realizado, no primeiro e quarto mês de lactação, com lactantes no município de Cuité, onde foram coletados amostras de leite humano, no primeiro e no quarto mês de lactação em visitas domiciliares. RESULTADOS: Obteve-se como resultado variação significativa nos itens proteína, lactose e valor calórico para o leite do primeiro mês de lactação em relação ao leite do quarto mês $(p<0,005)$. Para gordura, umidade, cinzas e acidez as concentrações não apresentaram variação significativa entre si nos meses estudados ( $p>0,005)$. CONCLUSÃO: Por fim, percebe-se que estudos deste seguimento se faz muito importante para o conhecimento a respeito da composição nutricional do leite materno, não somente para pesquisadores, mas também pelas próprias mães sobre a composição de seu leite.
\end{abstract}

Palavras chave: Lactação. Leite Humano. Composição de Alimentos. Análise de Alimentos.

ABSTRACT: OBJECTIVE: The present study aimed to analyze the composition of macronutrients, ash, moisture, acidity and energy density of human milk from mothers living in the municipality of Cuité-PB during the period 2012-2013. METHOD: This is a segment study carried out, in the first and fourth months of lactation, with lactating mothers in the municipality of Cuité, where samples of human

\footnotetext{
${ }^{1}$ Acadêmico do curso de Bacharelado em Nutrição da Faculdade Santa Maria, Cajazeiras-PB. E-mail: dpinheiro15@yahoo.com.br.

${ }_{2}^{2}$ Nutricionista. Mestre em Sistema Agroindustriais pela UFCG. Docente do curso de Nutrição da Faculdade Santa Maria - FSM.
} 
milk were collected, in the first and fourth months of lactation during home visits. RESULTS: A significant variation was found in the items protein, lactose and caloric value for milk in the first month of lactation in relation to milk in the fourth month ( $p$ <0.005). For fat, moisture, ash and acidity, the concentrations did not show significant variation between them in the months studied ( $p>0.005)$. CONCLUSION: Finally, it is clear that studies in this segment are very important for knowledge about the nutritional composition of breast milk, not only for researchers, but also for the mothers themselves about the composition of their milk.

Descriptors: Lactation. Milk, Human. Food Composition. Food Analysis. 


\section{INTRODUÇÃO}

$\mathrm{Na}$ espécie humana, o corpo da mulher prepara-se para a lactação desde os primeiros momentos da gravidez. O amadurecimento da glândula mamária ocorre a partir da puberdade, mas só durante a gravidez, pela elevação dos níveis circulantes dos hormônios ovarianos e placentários, é que o desenvolvimento mamário se vai completar, para dar início à produção de leite (WORTHINGTON- ROBERTS, 1988; NIVELLE et al., 2001).

Nos primeiros dias pós-parto as glândulas mamarias produzem o colostro, um líquido com densidade variável entre 1040 e 1060, com coloração amarelada, devido ao seu elevado teor de betacaroteno. Quando comparado ao leite maduro é mais viscoso, possuindo concentrações mais elevadas de proteínas, minerais e vitaminas lipossolúveis, particularmente $A, E$ e carotenóides, bem como menores teores de lactose, gorduras e vitaminas do complexo B (PRENTICE et al., 1987).

Alguns fatores podem influenciar, tanto na composição quanto no volume da secreção láctea, dentre eles, os genéticos, a nutrição materna, as técnicas de extração, o armazenamento e a administração ao bebê (CORRÍA, 2005). Em relação à composição dos nutrientes no leite humano, este depende da dieta e da capacidade de serem secretados pelo epitélio da glândula mamária. (AKRÉ, 1994; EUCLYDES, 2005; LAMOUNIER; LEÃO, 2000). Em relação à composição da secreção, para uma mesma mulher, são registradas variações no decorrer da lactação, ao longo do dia e durante uma mesma mamada, havendo diferenças entre o leite da frente e o último a sair (anterior e posterior) com alterações na concentração dos macro e dos micronutrientes (PICCIANO, 2001; EMMETT, P.M. \& ROGERS, I.S., 1997; VALDÉS et al., 1996).

O leite humano possui uma composição nutricional balanceada, na qual inclui todos os nutrientes essenciais (CALIL, 1991). Este é composto basicamente por proteínas, carboidratos, minerais, vitaminas e enzimas, sendo suficiente para suprir as necessidades das crianças, a Organização Mundial da Saúde preconiza 
aleitamento materno exclusivo até seis meses de vida, com a complementação de outros alimentos a partir do sexto mês até pelo menos dois anos de idade, devido a sua importância e seus benefícios (WHO, 2004).

Além da composição adequada de nutrientes, o leite materno possui outros componentes que atuam na defesa do organismo do lactente, como imunoglobulinas, fatores antiinflamatórios e imunoestimuladores (PASSANHA et al., 2010; MAHAN; ESCOTT-STUMP, 2010). Dentre a composição nutricional do leite, o conteúdo de gorduras é o que sofre a variação mais acentuada, onde sua concentração aumenta de forma progressiva desde o início até o final de cada amamentação. (CINGOLANI, 2004).

A lactose constitui o principal carboidrato do leite humano, estando presente em concentrações mais baixas no colostro do que no leite maduro. A lactose fornece ao redor de $50 \%$ do conteúdo energético total do leite materno. As gorduras constituem a maior fonte de energia do leite humano. Seu conteúdo no leite maduro varia entre 3 e $4 \mathrm{~g} / \mathrm{dl}$, correspondendo a aproximadamente 40 a $50 \%$ do total calórico; já o colostro possui concentração lipídica algo menor, em torno de 1,8 a 2,9 g/dl, que se eleva para valores intermediários $(2,9$ a $3,6 \mathrm{~g} / \mathrm{dl})$ no leite de transição. A necessidade proteica do recém-nascido de termo é estimada em cerca de 2,0 a 2,5 $\mathrm{g} / \mathrm{kg} / \mathrm{dia}$, decrescendo gradualmente até chegar a $1,3 \mathrm{~kg} / \mathrm{dia}$ por volta do quarto mês (CALIL, 1991).

Com isso, o conhecimento do seu perfil protéico, lipídico, de carboidratos e de minerais é de suma importância, já que estes preenchem todas as necessidades das crianças, principalmente nos seus primeiros meses de vida, sendo, portanto, imprescindíveis para promover um crescimento e desenvolvimento ótimos no recémnascido (ACCIOLY, 2009).

Fatores como as variações na composição, a importância nutricional e funcional do leite materno traz um grande interesse para seu estudo e análise (VELOSO, 2001). Desta forma, este estudo tem como objetivo analisar o valor calórico, os teores de umidades, cinza, o grau de acidez e a composição de macronutrientes do leite humano de mães nutrizes residentes no município de Cuité/PB no primeiro e quarto mês de lactação. 


\section{METODOLOGIA}

Trata-se de um estudo de seguimento cuja unidade amostral é comporta por mães lactantes residentes na zona urbana do município de Cuité - PB. Para conferir uma maior homogeneidade ao grupo estudado foram obedecidos critérios de inclusão, a saber: mães residentes na zona urbana do município de Cuité; mulheres em processo de amamentação e parto único; e lactentes com mais de 15 dias de parto; e critérios de exclusão, sendo estes: mães de recém-nascido retido em unidades de terapias intensivas neonatais ou berçários; crianças portadoras de anomalias congênitas que impedissem a amamentação; mães adolescentes e de bebês prematuros. As atividades referentes à pesquisa com leite humano e a sua manipulação foram aprovadas pelo Comitê de Ética em Pesquisa com Seres Humanos da Universidade Estadual da Paraíba, mediante o Processo de $n^{\circ}$ CAAE 0374.0.133.000-11.

\section{Captação da amostra}

A captação da amostra se iniciou com o levantamento da demanda de mulheres grávidas no nono mês de gestação e de mães de crianças no primeiro mês de vida existentes nas Unidades de Saúde da Família (UBS) da zona urbana do município de Cuité. O levantamento de dados foi referente ao período de setembro de 2012 a abril de 2013.

De acordo com este levantamento registrou-se 117 lactantes e gestantes em cinco Unidades de Saúde da Família da zona urbana pesquisadas. Destas mães, foram excluídas automaticamente 45 da pesquisa por não atenderem aos critérios de inclusão pré-estabelecidos, contudo houve mães que não foram encontradas e que não aceitarão participar da pesquisa, um número de 37 mães. Sendo assim, das 35 mães resultantes, 29 aderiram à pesquisa, 6 desistiram durante a coleta de amostras 
no primeiro mês de lactação, alegando diminuição no fluxo de leite materno, constituindo assim uma amostra de 23 lactantes no primeiro mês da coleta de dados, o que correspondeu a $19,66 \%$ das mães registradas nas USF do município de Cuité. $\mathrm{Na}$ realização da segunda coleta de dados em domicílios registrou-se uma perda de seguimento em 10 mães que informaram a inexistência da demanda de leite para a coleta de amostras, permanecendo assim 13 mães no quarto mês de lactação para coleta de dados.

\section{Coleta dos dados}

O procedimento de coleta de dados se iniciou com a abordagem da mãe no primeiro mês de lactação, em seu domicílio, com uma extensa explicação sobre os objetivos da pesquisa e os procedimentos para participação, e nos casos de adesão da mãe a pesquisa, esta assinou um Termo de Consentimento Livre e Esclarecido, sendo realizada a coleta de dados a partir de uma utilizando-se um questionário com informações socioeconômicas, de saúde e estado nutricional materna (peso e altura), assim como, informações pré-gestacionais, do lactente, características da gestação, parto, lactação e ordenha. No quarto mês foram colhidas mais algumas informações sobre o consumo do lactente, dados do ganho de peso da criança e aferido o peso da lactante. Os dados do questionário foram utilizados para outros fins de pesquisa.

Nas duas visitas eram realizadas coletas de leite materno, preferencialmente no período da manhã, sendo o conteúdo total da mama coletado em frasco de vidro âmbar, desmineralizado, estéril, fechado e identificado com o número do questionário e data da coleta. A quantidade sugerida para a coleta era em média de $150 \mathrm{~mL}$. A ordenha ocorreu de acordo com a preferência da mãe, podendo ser manual ou mecânica, neste último caso, o material era devidamente esterilizado. Depois da ordenha, as amostras eram armazenadas sob temperatura de congelamento (congelador doméstico), até a coleta das amostras.

Após coleto, as amostras foram acondicionadas em caixas isotérmicas com gelo e transferidas para o Laboratório de Físico-Química do Centro de Educação e 
Saúde da UFCG, onde passaram pelo processo de pasteurização $\left(65^{\circ} \mathrm{C} / 30 \mathrm{~min}\right.$.) e armazenamento em freezer sob temperatura de congelamento $\left(-18^{\circ} \mathrm{C}\right)$, até o momento da realização das análises de caracterização nutricional, preconizada pelo Instituto Adolfo Lutz (IAL, 2005).

\section{Análise de dados}

Foram realizados os seguintes procedimentos de análise: para análise de lipídios foi utilizado o método de Gerber, conforme metodologia proposta pelo Instituto Adolfo Lutz (IAL, 2005): a proteína bruta pelo método de Micro-Kjedahl, com o fator 6,38 multiplicado pela porcentagem de nitrogênio (AOAC, 2000) e o teor de lactose foi determinado pelo método de redução de Fehling (IAL, 2005).

Para a determinação da umidade procedeu-se a secagem da amostra, até obtenção de peso constante, a partir da estufa, com temperatura estabilizada a 105 ${ }^{\circ} \mathrm{C}$ por 24 horas consecutivas, segundo o Instituto Adolfo Lutz (IAL, 2005). O teor de resíduo mineral fixo, ou cinzas, foi determinado por carbonização seguida de incineração em mufla estabilizado a $550^{\circ} \mathrm{C}$, através da metodologia descrita pelo Instituto Adolfo Lutz (IAL, 2005). Acidez titulável em ácido láctico foi determinada por titulação com solução de hidróxido de sódio 0,1 N (IAL, 2005). Ao final foi realizada a conversão de acidez em ácido láctico em grau dornic $\left({ }^{\circ} \mathrm{D}\right)$, procedeu-se da seguinte forma, os valores encontrados em percentual foram multiplicados por 100.

O valor calórico foi calculado a partir dos teores da fração proteica, lipídica e de carboidratos, utilizando-se os coeficientes específicos que levam em consideração o calor de combustão 4,0; 9,0 e 4,0 kcal, respectivamente, conforme Dutra de Oliveira; Marchini (1998). Os resultados das análises físico-químicas do leite humano nos dois estágios de lactação foram submetidos à análise de variância (ANOVA), realizando-se teste de média de t-Student ao nível de $5 \%$ de significância $(p<0,05)$. Para o cálculo dos dados, utilizou-se o programa - Sigma Stat 3.1 (SIGMASTAT, 2009). 


\section{RESULTADOS E DISCUSSÃO}

A composição média obtida para o leite humano nos diferentes estágios de lactação é apresentada na Tabela 1. Em seus resultados ocorreu variação significativa nos itens proteína, lactose e valor calórico para o leite do primeiro mês de lactação em relação ao leite do quarto mês $(p<0,005)$. Para gordura, umidade, cinzas e acidez as concentrações não apresentaram variação significativa entre si ( $p$ $>0,005)$.

Com relação às características físico-químicas, foi dada ênfase a acidez em ácido láctico que é um parâmetro qualitativo e apresentou um valor médio de $5^{\circ} \mathrm{D}$ no primeiro mês de lactação e de $6^{\circ} \mathrm{D}$ no quarto mês de lactação. Segundo a RDC 171, de 4 de setembro de 2006 o limite estabelecido para grau de acidez em leite humano é inferior a $8^{\circ} \mathrm{D}$. Esta investigação foi realizada pelo fato de que a acidificação do leite pode levar à redução dos componentes nutricionais - principalmente a diminuição da biodisponibilidade do cálcio e fósforo presentes e imunológicos e desqualificar sua utilização (NOVAC; CORDEIRO, 2007). Alguns fatores são apontados como determinantes, como: composição química do leite, crescimento microbiano, temperatura de armazenamento e, possivelmente, a alimentação da nutriz (CAVALCANTE, 2005; ICHISATO, SHIMO, 2001; NOVAK; CORDEIRO, 2007). Com relação aos valores de umidade e cinzas ambos demonstram-se inferiores aos corroborados por Silva (2007). 
Tabela 1 - Caracterização físico-química e composição nutricional média de amostras de leite materno de mães residentes na zona urbana do município de Cuité, 2012/2013.

\section{PERIODO DE COLETA}

\section{COMPOSIÇÃO CENTESIMAL DO LEITE MATERNO $\quad 1^{\circ}$ Mês $\quad 4^{\circ}$ Mês}

\begin{tabular}{ccc}
\hline UMIDADE \% & $87,85 \pm 0,016$ & $88,99 \pm 0,18$ \\
CINZAS \% & $0,16 \pm 0,03$ & $0,16 \pm 0,03$ \\
GORDURA \% & $3,08 \pm 0,00$ & $2,45 \pm 0,00$ \\
PROTEÍNA \% & $1,21 \pm 0,12^{*}$ & $0,68 \pm 0,10$ \\
LACTOSE \% & $6,55 \pm 0,07^{*}$ & $5,99 \pm 0,04$ \\
ACIDEZ ( ${ }^{\circ}$ D) & $5 \pm 0,00$ & $6 \pm 0,0$ \\
CALORIAS (Kcal/100 mL) & $58,80 \pm 7,65^{\star}$ & $48,75 \pm 9,93$ \\
\hline
\end{tabular}

${ }^{*}$ Diferença estatística ao nível de $5 \%$ de probabilidade no teste t-Student.

Observando à composição nutricional das amostras de leite analisadas no primeiro mês de lactação, pode-se constatar que o valor médio de lactose obtido de $6,55 \%( \pm 0,07)$ condiz com a média proposta por Silva $(2007)$ que é de $7,5 \%( \pm 0,66)$ para o leite maduro e com o estudo de Calil (2003), que propõe que a lactose possui uma composição que oscila entre $7 \mathrm{~g} / \mathrm{dL}$ no leite maduro. Em comparação com o estudo de Bortolozo (2004) o valor de lactose $8,60 \mathrm{~g}( \pm 0,93)$, o valor encontrado na pesquisa encontra-se inferior a este estudo e com o de Euclydes (2005) que foi na média de $7,4 \mathrm{~g}$ de lactose. Já no quarto mês o valor obtido de lactose $5,99 \%( \pm 0,04)$, se encontra abaixo da média encontrada nestes estudos. Destaca-se que a lactose é o principal carboidrato do leite humano e deve fornecer $42 \%$ das necessidades energéticas do bebê. $\mathrm{Na}$ literatura não possui estudo que cita se há diferença significativa entre os teores de lactose nos diferentes estágios do leite humano.

Os teores de proteína obtidos na presente pesquisa de primeiro mês 1,21\% $( \pm 0,12)$ mostraram-se semelhantes aos encontrados por Silva (2007), Bortolozo (2004), Euclydes (2005) e Almeida (2008) que é de 1,12\% $( \pm 0,28), 1,07 \mathrm{~g}( \pm 0,22)$, $1,17 \mathrm{~g}$ e $1,25( \pm 0,14)$, respectivamente. $O$ valor médio do quarto mês se encontra inferior aos valores obtidos nestes estudos supracitados. A concentração de proteína do leite materno decresce rapidamente no primeiro mês de lactação e mais 
lentamente a partir daí, acompanha a velocidade de crescimento da criança (EUCLYDES, 2005).

A quantidade de lipídios encontrada no primeiro mês e no quarto mês, assemelha-se ao encontrada por Bortolozo (2004) de 2,56g $( \pm 0,79)$ e por Almeida (2008) de 2,7\% $( \pm 1,5)$, e ainda superior aos expostos por Silva (2007) de 1,38\% $( \pm 0,60)$ e inferiores aos citados por Accioly et al. (2009) e por Euclydes (2005). Como pode ser observado há uma incoerência entre os estudos principalmente em relação ao teor de gordura, tendo em vista que é o componente mais variável do leite humano, e esta variação entre os valores encontrados também podem ser justificadas em decorrência do horário da coleta, da possível não retirada do leite posterior pela lactante, como também podem estar relacionados à adesão de moléculas de gordura nas paredes internas dos recipientes utilizados e formação de cristais de gelo durante o congelamento (SILVA, et al. 2007; BORGO, 2011).

Recomenda-se que crianças até dois meses de idade devam ingerir em média $520 \mathrm{Kcal} /$ dia (BRASIL, 2002). No presente estudo o valor calórico do leite resultado assemelha aos estudos de Euclydes (2005), Silva (2007) e Bortolozo (2004).

A falta de estudo que aborde a composição nutricional do leite humano especificando em que período de lactação ele se encontre dificulta a real comparação dos dados, uma vez que os estudos referenciados utilizam em sua metodologia apenas do uso leite maturo, e como os resultados da pesquisa observou-se que há mudanças de sua composição de acordo com estágio de lactação do primeiro para o quarto mês de lactação.

De suma importante destacar que diversos fatores podem ser determinantes para este resultado como às técnicas de coleta de dados e os procedimentos de conservação da amostra de leite humano utilizados no presente estudo.

É necessário considerar, entretanto, que a interferência dos esquemas de amostragem, nem sempre reflete o conjunto da produção láctea no decorrer de um dia, e pode ser causa de resultados que venham a sub ou superestimar as taxas de secreção verificadas, e o desempenho na lactação, dissimulando as possíveis influências da nutrição materna no leite, assim como as metodologias que apresentem variações, no que concerne não somente às determinações, mas também à forma de interpretação dos resultados (VILLALPANDO et al., 1991). 
Em relação ao desmame precoce uma das causas resultantes é o desconhecimento da mãe em relação à qualidade de seu leite, não somente para sanar a fome, mas também para conduzir a um bom desenvolvimento do seu bebê (DIAS, 2010). A partir disso ao final das análises foi elaborado um rótulo nutricional, que foi entregue as nutrizes, contendo os dados das análises do seu leite durante o primeiro e o quarto mês de lactação. Esta medida foi uma estratégia para mostra a elas a composição nutricional do seu leite e que ele é um alimento completo que contém proteína, carboidrato, lipídio e valor energético igual a qualquer outro alimento é em sua composição teores de umidade e cinzas. Serviu também para desmistificar para muitas delas de que o leite é um alimento pobre e não contém os nutrientes necessários para o crescimento da criança.

\section{CONCLUSÃO}

Com base nos resultados físico-químicos conclui-se que a acidez encontravase dentro dos padrões aceitáveis em ambos os períodos de lactação estudados. Quando comparamos com os valores médios dos macronutrientes obtidos no primeiro e quarto mês de lactação destaca-se uma diferença significativa nos valores de proteína, lactose e valor calórico para o leite do primeiro mês de lactação que possa ser explicado devido à forma pela qual as amostra foram coletadas, se foi alcançado o leite posterior e a composição da dieta das nutrizes que contribuí, assim como seu estado nutricional, na composição nutricional do leite materno e nos dados estatísticos. Contudo os valores encontrados se encontram semelhante a alguns estudos realizados utilizando o leite humano maduro.

Estudos como este demostram a qualidade nutricional do leite materno e ressalta a necessidade de orientações dispensadas a nutrizes sobre o aleitamento não apenas para incentivar a prática, mas para permitir a sua realização correta garantindo a criança um leite com melhores propriedades nutricionais e se faz muito importante para o conhecimento a respeito de sua composição, não somente para pesquisadores, mas também pelas próprias mães sobre a composição de seu leite. 


\section{REFERÊNCIAS BIBLIOGRÁFICAS}

ACCIOLY, E.; SAUNDERS, C.; LACERDA, E. Ma . A. Nutrição em obstetrícia e pediatria. 2ed. Rio de Janeiro: Cultura Médica: Guanabara Koogan, 2009.

ALMEIDA, S. D. S. Contagem celular somática, bacteriana total e composição do leite humano. Dissertação (Mestrado em ciência animal), Escola de Veterinária, Universidade Federal de Goiás, 2008.

AKRÉ, J. Alimentação infantil: bases fisiológicas. Trad. Anna Velochko e Tereza Toma. São Paulo: IBFAN Brasil; 1994.

AOAC. Official Methods of Analysis.14.ed. Ass. Off. Analytical.Chem.Washington, USA, 2000.

Brasil. Ministério da Saúde. Organização Pan-Americana da Saúde. Guia alimentar para crianças menores de dois anos. Serie A. Normas e manuais técnicos. Brasília, DF: Ministério da Saúde. 2002.

BORTOLOZO, E. A. F. Q.; TIBONI, E. B.; CÂNDIDO, L. M. B. Leite humano processado em bancos de leite para o recém-nascido de baixo peso: análise nutricional e proposta de um novo complemento. Rev Panam Salud Publica/Pan Am J Public Health 16(3), 2004.

CALIL, V.M.L.T.; LEONE, C.R.; RAMOS, J.L.A. Composição nutricional do colostro de mães de recém nascidos de termo adequados e pequenos para a idade gestacional e composição nutricional do leite humano nos diversos estágios da lactação, vantagens em relação ao leite de vaca. Abril de 1991

CALIL, Valdenise Martins Laurindo Tuma; FALCÃO, Mario Cícero. Composição do leite materno: o alimento ideais. Rev Med, jan-dez. v.82 n.1-4, p. 1-10, 2003.

CAVALCANTE, J. L. P. et al. Uso da acidez titulável no controle de qualidade do leite humano ordenhado. Ciênc. Tecnol. Alim. v.25, n.1, p.103-108, 2005.

CINGOLANI, Horácio E. Fisiologia humana de Houssay. 7. ed. Atualizada e ampliada. Porto Alegre, RS: Artmed, 2004.

CORRÍA, V. D. A. R. Lactancia materna: evaluación nutricional em El reciénnacido. 21 Revista Cubana Pediátrica, v. 77, n. 2, p. 1-10, 2005.

DIAS, M. C. A. P.; FREIRE, L. M. S.; FRANCESCHINI, S. C. C. Recomendações para alimentação complementar de crianças menores de dois anos. Revista de Nutrição. Campinas SP, 23(3):475-486, 2010.

EMMETT, P.M.; ROGERS, I.S. Properties of human milk and their relationship with maternal nutrition. Early Hum. Dev., v. 49, p. 7-28, 1997.

EUCLYDES, M.P. Nutrição do lactente: base científica para uma alimentação adequada. 2.ed. rev. atual. Viçosa, MG, 2000. 488p.

EUCLYDES, M.P. Nutrição do lactente, base científica para uma alimentação saudável, 3 . ed. Viçosa: Suprema, 2005. 548p.

ICHISATO, S. M. T.; SHIMO, A. K. K. Aleitamento materno e as crenças alimentares. Rev. Lat. 
Am. Enferm. v.9, n.5, p.70-76, 2001.

INSTITUTO ADOLFO LUTZ. Normas analíticas de Instituto Adolfo Lutz. 4. ed. São Paulo, v. 1, 2005. $1018 \mathrm{p}$.

LAMOUNIER, J. A.; LEÃO, E. Nutrição na infância. In: DUTRA-DE-OLIVEIRA, J. E.; MARCHINI, J. S. Ciências nutricionais. (eds.). São Paulo: Sarvier, 2000; 403p.

MAHAN, L. K.; ESCOTT-STUMP, S. Krause: Alimentos, Nutrição e Dietoterapia.12a edição. Rio de Janeiro: Elsevier, 2010.

NEVILLE, M.C. e MORTON, J. (2001). Physiology and endocrine change underlying human lactogenesisIl. In J. Nutri. 131, pp. 3005S-8S.

NOVAK, F. R.; CORDEIRO, D. M. The correlation between aerobic mesophilic microorganism counts and Dornic acidity in expressed human breastmilk. J. Pediatr. v.83, n.1, p.87-91, 2007.

PASSANHA, A.; CERVATO-MANCUSO, A. M.; SILVA, M. E. M. P. Elementos protetores do leite materno na prevenção de doenças gastrintestinais e respiratórias. Rev. Bras. Cresc. e Desenv. Hum. 2010; 20(2): 351-360.

PRENTICE, A. et al. - The nutritional role of breast-milk IgA and lactoferrin. Acta Paediatr. Scand. 76: 592, 1987.

PICCIANO, M.F. Nutrient composition of human milk. Pediatr. Clin. North Am., v. 48, n. 1, p. 53-67, 2001.

SIGMASTAT (programa de computador). Versão 3.1. Point Richmond (Califórnia): Comercial; 2009.

SILVA, R. C; GIOIELLI, L. A.; ESCOBEDO, J. P. Composição centesimal do leite humano e caracterização das propriedades físico-químicas de sua gordura. Quim. Nova, vol. 30, No.7, 1535-1538, 2007.

VALDÉS, V.; SÁNCHEZ, A.P.; LABBOCK, M. Manejo Clínico da Lactação. Assistência à Nutriz e ao Lactente. Rio de Janeiro: Revinter, 1996.

VELOSO, Ana Cristina A.; TEIXEIRA, Natércia; FERREIRA, Isabel M.; FERREIRA, Margarida A. Metodologias de doseamento das proteínas do leite. Boletim de Biotecnologia. 2001.

VILLALPANDO, S.; SANTIAGO, S.; FLORESHUERTA, S. Mater nal nutritional status and milk volume. Is ther e a cause- ef fect relationship? Archiv. Latin. Nutr. v. 41. n. 3. p. 293-03. 1991.

World Health Organization. Evidence for the ten steps to successful breastfeeding Rev.ed. Division of Child Health and Development. 2004.

WORTHINGTON- ROBERTS (1988). Lactação e leite humano: considerações nutricionais. In: WORTHINGTON- ROBERTS, B.S, VERMEERSCH, J. e WILLIWAMS, S.R. Nutrição na gravidez e lactação. $3^{\mathrm{a}}$ ed. Rio de Janeiro, Editora Guanabara. pp. 187-240. 